ISSN: 0212-5374

DOI: http://dx.doi.org/10.14201/et2015331163192

\title{
LA DEMOSTRACIÓN EN LAS MATEMÁTICAS. UN EJEMPLO DE APLICACIÓN EN EL AULA CON ALUMNOS DE $3 .^{\circ}$ ESO
}

\author{
The role of Matematical demostrations. A sample \\ application with $3^{\text {rd }}$ ESO students in the classroom
}

\section{Les démonstrations mathématiques. Un exemple \\ d'application avec les étudiants dans la classe de $3^{\text {eme }}$ ESO}

Enrique SÁnChez Freire* y Juan Antonio Gil PASCUAL**

* IEs Luis García Berlanga. Correo-e: enrique_sanchez_freire@yaboo.es

** Sociedad Española de Investigación en Educación Matemática (SEIEM).

Correo-e: jgil@edu.uned.es

Recibido: 10-02-2014; Aceptado: 18-04-2014; Publicado: 30-04-2015

BIBLID [0212-5374 (2015) 33, 1; 163-192]

Ref. Bibl. ENRIQUE SÁNCHEZ FREIRE y JUAN ANTONIO GIL PASCUAL. La demostración en las Matemáticas. Un ejemplo de aplicación en el aula con alumnos de 3. ${ }^{\circ}$ ESO. Enseñanza \& Teaching, 33, 1-2015, 163-192.

RESUMEN: En este artículo mostramos la influencia que han tenido el rigor y sus respectivos procedimientos en diferentes momentos de la Historia de las Matemáticas y como algunas demostraciones y problemas han jugado un papel importantísimo en el desarrollo de las Matemáticas. Complementaremos esta fundamentación teórica con los resultados de una experiencia de aula en donde comprobaremos que un grupo en donde se aplicó una metodología en la cual los procesos de argumentación y demostración tuvieron una especial relevancia obtuvo mejores resultados en unas pruebas de resolución de problemas que otro grupo del mismo nivel al que se le aplicó una metodología más tradicional. 
Palabras clave: educación matemática; didáctica de las matemáticas; educación secundaria; demostraciones; experiencia en el aula.

SUMMAY: In this article we show the influence of mathematical accuracy and its procedures through different periods of Mathematical History and the role played demonstrations and problem solving in the development of Mathematics. We will complete this theoretical dissertation with some experimental data obtained in the classroom. We will compare the results obtained by a class group following a teaching methodology based upon mathematical accuracy, reasoning and demonstration versus a class group using a more traditional teaching approach. The comparison of the two groups shows how the first group obtained better results in tests of mathematical problem solving.

Key words: education; didactics; mathematics; secondary education; mathematical proofs; class experience.

RÉSUMÉ: Dans ce article nous montrons l'influence que a eu la rigueur mathématique et les processus associes au raisonnement dans les différents moments de l'Histoire de la mathématique. Nous monstrons comment certaines démonstrations et problèmes ont joué un rôle très important dans le devenir de la Science Mathématique. Nous complèterons cette explication théorétique avec les résultats d'une expérience de classe. Nous vérifierons que un groupe où il était appli une méthodologie basé sur l'utilisation de las démonstrations et le raisonnement a obtenu des meilleiurs résultats dans les preuves de résolutions des problèmes qu'ils ont réalisé. La comparaison des deux groupes montre clairement la différence.

Mots clés: éducation; didactique; mathématique; éducation secondaire; démonstrations mathématiques; expérience de salle de classe.

\section{LA DEMOSTRACIÓN Y EL RIGOR EN LAS MATEMÁTICAS}

Las justificaciones, argumentaciones y las demostraciones son procedimientos que han estado siempre inherentes al quehacer matemático. Aunque los primeros indicios en el desarrollo de las Matemáticas aparecieron en las civilizaciones egipcia y babilónica con alguna prueba sobre relaciones de determinadas magnitudes geométricas, parece ser que existe unanimidad en indicar que la verdadera génesis de la demostración tuvo lugar en la Grecia Clásica.

Según Bombal (2010) las primeras demostraciones se le atribuyen en el siglo vi a. C. a Tales de Mileto. Sus biógrafos indicaron que era un gran entusiasta del rigor y a él se le atribuyen las demostraciones de que un diámetro divide a un círculo en dos partes iguales, los ángulos de un triángulo suman dos rectos y un ángulo inscrito en un semicírculo es recto. 
En el siglo vi a. C. Pitágoras, un discípulo de Tales, se centró en las relaciones precisas de diferentes fenómenos que se presentaban en la naturaleza. La asunción de que todo objeto estaba formado por una colección de átomos individuales e indivisibles le condujo a pensar que todas las magnitudes eran conmensurables, es decir, existía una medida común para ambas. Este hecho permitió a la Escuela Pitagórica facilitar la visualización de algunos resultados geométricos, convirtiéndolos en aritméticos, y desarrollar numerosos resultados de Geometría Plana.

El punto de inflexión se situó en el siglo v a. C. cuando se descubrieron magnitudes inconmensurables como la diagonal y el lado de un pentágono regular, y la irracionalidad de $\sqrt{2}$. En estos momentos se produjo la conocida como primera crisis en los fundamentos de las Matemáticas ya que los matemáticos de la época se dieron cuenta de que no siempre se podía trabajar aritméticamente en Geometría y que los objetos matemáticos no son tan simples como podían parecer. En la línea de lo expuesto por Arsac (1987), parece ser unánime la opinión de que estos hechos supusieron el verdadero origen de la demostración. El estudio de estos problemas, tanto en el marco aritmético como en el geométrico, unido a las paradojas del infinito de Zenón, condujo a la creación del método axiomático deductivo en la segunda mitad del siglo v a. C.

Uno de los ejemplos más claros lo encontramos en Los Elementos de Euclides. Partiendo de una serie de verdades evidentes o axiomas (en este caso 23 definiciones, 5 postulados y 5 nociones comunes) y utilizando las leyes de la Lógica deductiva se van construyendo nuevos resultados. Este sigue siendo el método utilizado actualmente, con la diferencia de que hemos variado las condiciones iniciales. Los métodos utilizados por Euclides en su obra fueron el germen de la nueva Matemática. Sólo Hilbert, tras más de 2000 años, fue capaz de percibir la omisión (no de errores) de la justificación de ciertos resultados en su método.

El apogeo de las Matemáticas griegas llegó en el siglo III a. C. con Arquímedes. Tras él, las Matemáticas en general sufrieron un estancamiento en su fundamentación teórica que duró alrededor de 18 siglos. La principal causa de este declive se debe a que no pudieron formalizar en su sistema el concepto de infinito, lo que les produjo una autolimitación en la producción de resultados.

La herencia griega fue trasmitida por toda Europa, primero por el Imperio Bizantino y posteriormente por los árabes. Estos últimos enriquecieron algunos procesos aritméticos y algebraicos. Por ejemplo, resolvieron la ecuación de segundo grado con técnicas geométricas.

En el siglo XIV se intentaba solucionar problemas ligados a situaciones reales (estudios de movimientos, problemas geográficos, militares, etc.) ignorando posibles trabas lógicas que podían producirse con el uso de conceptos relacionados con el infinito y dando prioridad a la generación de resultados. Estas ideas continuaron en el Renacimiento con matemáticos como Kepler y Cavalieri y sus técnicas de los indivisibles para calcular volúmenes. Eran conscientes de la falta de rigor de sus procedimientos, pero justificaban sus métodos por los extraordinarios resultados que obtenían. De hecho, a Cavalieri se le atribuye la frase 
"el rigor es asunto de los filósofos, más que de los matemáticos». En esta misma época, el desarrollo simbólico que se produjo le permitió a la escuela italiana obtener fórmulas de resolución de ecuaciones de tercer y cuarto grado mediante radicales. Este sería uno de los principales hilos conductores del posterior desarrollo del Álgebra.

En el siglo XVII el estudio de la relación entre los problemas de la tangente a una curva y el área encerrada bajo una curva trajo consigo la introducción de nuevos métodos que propiciaron la invención del Cálculo. El cambio de mentalidad lo iniciaron Descartes y Fermat al transformar los métodos geométricos de expresar magnitud como suma de indivisibles en técnicas aritméticas de sumación de series infinitas, sentando de este modo las bases de la Geometría Analítica. En este proceso de aritmetización se creó un ente abstracto fundamental: el infinitésimo. Las técnicas de manipulación con este ente culminaron con la invención del Cálculo llevada a cabo por Newton y Leibniz.

La importancia de la invención del Cálculo, tanto en las Matemáticas, como en su aplicación a situaciones reales, era sin ningún lugar a dudas espectacular, pero esta espectacularidad contrastaba con la debilidad de los fundamentos lógicos en los que se sustentaba. Esta cuestión fue objeto de preocupación por parte de algunos matemáticos de la época, entre los que se encontraba el propio Leibniz, aunque en absoluto se consideraba una cuestión primordial.

En el siglo XVIII nos encontramos con Euler, uno de los matemáticos más prolíficos de la historia debido a que en sus métodos dejó de lado en gran medida el rigor. De hecho, él considera una demostración, más que una verdad matemática incontestable, como una predicción con una alta fiabilidad, corroborada con un número suficiente de casos particulares. Esta idea no era compartida por la totalidad de la comunidad matemática, lo que hizo que existieran muchas disputas por la veracidad de ciertos resultados y que se produjeran multitud de contraejemplos sobre los resultados que iban apareciendo, produciéndose de este modo una inseguridad global. Un ejemplo de esto lo tenemos en los logaritmos de números negativos. Mientras Leibniz sostenía que eran imaginarios basándose en trabajos de series divergentes, Johann Bernouilli defendía que $(-n)=\log (n)$, ya que

$$
2 \cdot \log (-n)=\log (-n)^{2}=\log n^{2}=2 \log n
$$

Las inconsistencias lógicas que producía el uso de infinitésimos tenían grandes detractores, entre los que se puede citar a Berkeley. Acusó a los seguidores de Newton y Leibniz de utilizar métodos que no comprendían, basados en inconsistencias lógicas y en conceptos ambiguos. Consideraba que algunos de los resultados a los que llegan pueden ser correctos como consecuencia de compensación de errores. Esta idea sería repetida por matemáticos como Lagrange o Maclaurin que buscaron con posterioridad una justificación a la fundamentación del Cálculo.

En los inicios del siglo XIX tuvo lugar una situación inevitable: la revisión científica de los resultados. La revisión de los fundamentos del Cálculo la inició Cauchy, basando su desarrollo en su definición de infinitésimo, variable con límite 
cero. Sobre este concepto elaboró los conceptos básicos de la Teoría de Funciones, define la derivada como el cociente del límite de incrementos y construye, ya de forma rigurosa, todo el Cálculo. La obra de Cauchy la culmina Weiertrass definiendo formalmente el concepto de límite en términos de $\boldsymbol{\varepsilon}-\boldsymbol{\delta}$, que permitiría la construcción del Cálculo en función de las propiedades del sistema de números reales. Todo este proceso hace que vaya variando paulatinamente el concepto de rigor y de lo que es y deja de ser un paso trivial. En esta situación, los matemáticos de este siglo trataron de encontrar ya no sólo la fundamentación rigurosa del Cálculo, sino de toda la Matemática.

Se comenzaron a encontrar relaciones entre resultados enmarcados en teorías distintas, lo que hizo que se relegara la importancia de la naturaleza de objeto, en favor de las relaciones con otros objetos y que de este modo aparecieran las estructuras algebraicas básicas. El nacimiento de estas y otras nuevas teorías no hacía más que aumentar la inestabilidad de la comunidad matemática porque nadie garantizaba que los nuevos resultados que se iban generando estuvieran sustentados en unos cimientos estables desde el punto de vista lógico. Había que aclarar las reglas del juego para terminar con las contradicciones y para ello se volvió a recurrir al método axiomático griego.

Durante un tiempo se pensó que la Lógica y la Teoría de Conjuntos podían ser esos cimientos estables, pero las paradojas que surgieron en esta teoría hicieron que también tuviera que axiomatizarse. Esto llevó a Hilbert a proponer fundamentar la Matemática con métodos finitistas. Propuso utilizar la lógica simbólica para crear un lenguaje artificial, siendo muy cuidadoso al establecer sus reglas, de modo que no surgieran contradicciones. La idea de Hilbert era crear para el razonamiento, la deducción y las Matemáticas un lenguaje artificial perfecto. Por ello hizo especial hincapié en la importancia del método axiomático de la Grecia Clásica. Pretendía establecer unas reglas tan precisas mediante las cuales toda demostración pudiera someterse a lo que él denominó procedimiento mecánico que pudiera afirmar si la demostración era o no correcta. En ningún momento pensó que la forma de trabajar en Matemáticas fuera de este modo, sino que, si fuera posible esta teoría, se podría utilizar para ver cuál es el verdadero potencial de las Matemáticas y hasta dónde podría llegar.

Las ilusiones de Hilbert se vinieron abajo cuando Gödel demostró en 1931 que su idea fallaba incluso si nos hubiésemos centrado únicamente en la Aritmética elemental consistente en los números naturales con las operaciones de la adición y el producto. Cualquier sistema formal que trate de tener toda la verdad y nada más que la verdad sobre las Matemáticas (o incluso sobre la Aritmética elemental) será o incoherente o incompleto.

El siguiente avance relevante sobre el tema ocurrió cinco años después en Inglaterra cuando Turing demostró la no computabilidad. Recordemos que Hilbert pensaba que debía existir un procedimiento mecánico que verificase si una demostración era o no era correcta. Aunque Hilbert nunca llegó a aclarar con precisión su idea de procedimiento mecánico, Turing entendió este procedimiento a través 
de lo que hoy denominamos una máquina de Turing, que es un modelo computacional que realiza una lectura/escritura de forma automática sobre una entrada y generando una salida, basándose su funcionamiento en una función de transición. A pesar de su sencillez, el invento de Turing tiene una característica muy importante, que es que puede realizar cualquier cómputo que pueda realizar un ser humano. Ante esta situación Turing se preguntó: ¿qué le sería imposible a esta máquina? En poco tiempo encontró respuesta a su pregunta en el problema de la detención. Este problema consiste en decidir de antemano si una máquina de Turing (o un programa de ordenador) acabará por hallar su solución deseada y, por tanto, se detendrá. Este problema es de fácil solución si se impone un límite de tiempo, pero en caso contrario no es tan sencillo. Lo interesante de todo esto es un corolario que nos indica que si no hay de antemano una forma de determinar mediante cálculos si un programa va a detenerse o no, tampoco puede haberla mediante razonamientos lógicos. El merito de Turing fue que demostró que ningún sistema axiomático formal puede ser completo.

El siguiente paso lo dio Chaitin (2003) cuando sospechó que el motivo de la incompletitud podía deberse a la aleatoriedad característica de muchos resultados matemáticos. Relacionando el concepto de aleatoriedad con conceptos físicos como la entropía, consiguió relacionar el grado de desorden de una determinada situación con el tamaño del programa de ordenador más sencillo capaz de describirla. Pero esta idea nos conduce a un hecho perturbador: allí donde miremos encontraremos incompletitud. Esto se debe a que no podemos asegurar que el programa que tenemos delante sea el de menor tamaño posible, es decir, el más sencillo. Esta tarea escapa al razonamiento matemático.

Si trasladamos estas ideas a las Matemáticas, se puede observar que mientras los axiomas son finitos y bastante concisos, los resultados matemáticos abarcan un tamaño descomunal. Analizado desde este punto de vista, el teorema de incompletitud de Gödel no es ni misterioso ni complicado, sino más bien natural y casi se podría decir que evidente, ya que los axiomas solo pueden abarcar una cantidad finita de información.

Para cuadrar todas estas ideas faltaría poner un ejemplo concreto de un resultado matemático que se escape del razonamiento lógico. Durante muchos años, se tomó como ejemplo el último teorema de Fermat que dice que para cualquier número natural $n>2$, no existen tres enteros $a, b$ y $c$, no nulos, que verifiquen que $a^{n}$ $+b^{n}=c^{n}$. Desde 1637 en que Fermat conjeturó este teorema (aunque él asegura que también encontró una demostración) hasta 1995 que fue cuando Wiles lo demostró, este resultado fue considerado como unos de los mayores retos matemáticos y durante parte del siglo xx fue considerado además como aquel ejemplo de resultado que se escapa al razonamiento lógico. En la actualidad hay muchas conjeturas abiertas que no han sido aún demostradas, pero esto no quiere decir que sean ejemplos de proposiciones matemáticas indecidibles de demostrar si son ciertas o falsas.

Se puede observar como el entendimiento de la demostración del último teorema de Fermat realizada por Wiles está al alcance de muy pocos matemáticos, y 
es que las Matemáticas han llegado en la actualidad a un nivel de desarrollo y especialización que los nuevos resultados que van surgiendo los tenemos que asumir más bien por un acto de fe tras la aprobación de un conjunto de expertos en la materia. Esto se observa en la definición de demostración de Balacheff (2000):

Se trata de una serie de enunciados que se organizan siguiendo un conjunto bien definido de reglas. [...] Una demostración se convierte en tal, después del acto social de "aceptar que lo es" (p. 13).

\section{LA DEMOSTRACiÓN Y LA RESOlUCiÓN DE PROBLEMAS COMO HERRAMIENTA DE DESARROLLO EN LAS MATEMÁTICAS}

Desde un punto de vista histórico, tanto los retos que han supuesto la realización de diferentes demostraciones como la resolución de algunos problemas han sido, y de hecho siguen siendo, la mayor fuente de inspiración para la obtención de nuevos conocimientos y técnicas matemáticas.

Lo que haremos en este punto será citar solo algunos momentos claves en la historia de las Matemáticas en los que la resolución de un determinado problema o la realización de una determinada demostración supusieron una evolución en el desarrollo de esta ciencia.

Si nos remontamos al siglo v a. C. los pensadores griegos de la época, entre los que podemos destacar a Anaxágoras e Hipócrates, propusieron una multitud de problemas de índole geométrica. De todos ellos han destacado tres por su perduración a lo largo del tiempo. Se conocen como los tres problemas clásicos y son la cuadratura del círculo, la trisección del ángulo y la duplicación del cubo. Los enunciados de estos problemas son los siguientes:

- Cuadratura de un círculo. Dado un círculo, construir únicamente con regla y compás un cuadrado con igual área que el círculo inicial.

- Trisección de un ángulo. Dividir un ángulo cualquiera en tres partes iguales utilizando únicamente regla y compás.

- Duplicación de un cubo. Dado un cubo, construir, utilizando únicamente regla y compás, otro cubo que duplique el volumen del cubo inicial.

El estudio de estos tres problemas ha sido abordado a lo largo de la historia desde técnicas muy diversas, cuestión esta que ha contribuido al desarrollo de las Matemáticas. No fue hasta el año 1837 cuando Wantzel demostró que los problemas de la trisección del ángulo y la duplicación del cubo no tenían solución tal y como estaban planteados originalmente. El problema que más se resintió fue el de la cuadratura del círculo. Se intentó resolver desde las técnicas elementales de la Geometría Plana, utilizando técnicas algebraicas, herramientas trigonométricas, series infinitas, etc. Finalmente fue resuelto utilizando las técnicas que nos proporcionaba el Análisis Matemático. Fue Lindemann el que probó que este problema también era irresoluble utilizando únicamente la regla y compás. Para 
ello tuvo que probar que el número $\pi$ es trascendente, es decir, que no es raíz de ningún polinomio con coeficientes enteros. Esto nos da una idea de cómo la resolución de un problema geométrico con una apariencia sencilla debe requerir del desarrollo de otro tipo de herramientas para su resolución, fuera incluso de la propia área en la que se encuadró el problema original.

A pesar de que estos problemas no tienen solución tal y como están planteados, los propios griegos de la época los resolvieron pero sin la restricción de utilizar únicamente la regla y el compás, creando para ello curvas que, aparte de resolver parcialmente estos problemas, ayudaron a desarrollar las Matemáticas y otras ramas científicas.

Un ejemplo muy claro de esto lo encontramos en la espiral de Arquímedes, que es el lugar geométrico de un punto moviéndose con una velocidad constante sobre una semirrecta que gira sobre el punto de origen a una velocidad constante.

FIGURA 1

Espiral de Arquímedes

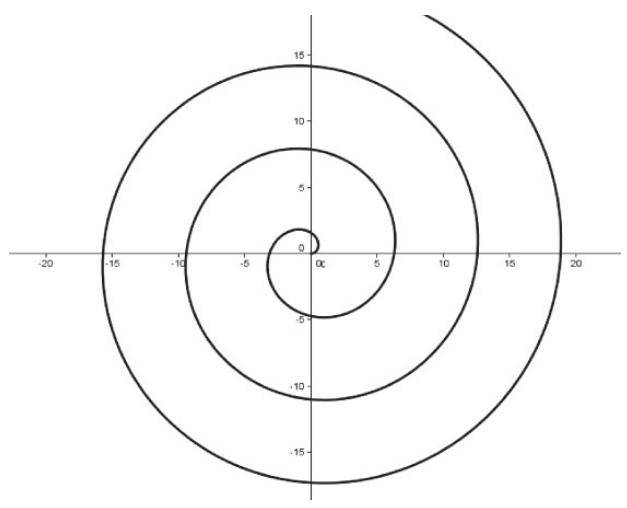

FIGURA 2

Trisección de un ángulo con la espiral de Arquímedes

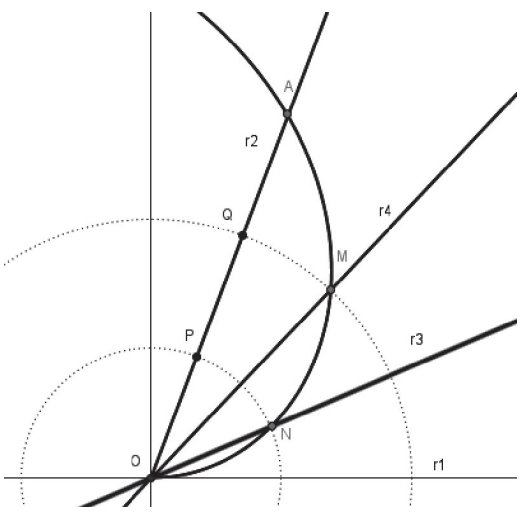

Esta espiral tiene como característica que la separación entre las distintas vueltas es constante, no como sucede en la otra espiral famosa, la espiral logarítmica, en donde las separaciones entre las vueltas forman una progresión geométrica.

Utilizando las propiedades de esta espiral se puede trisectar un ángulo y cuadrar un círculo. La cuestión no es complicada teniendo en cuenta que la espiral de Arquímedes tiene por ecuaciones paramétricas $\alpha(t)=(t \cdot \cos t, t \cdot \operatorname{sen} t)$, con $t \in[0,+\infty)$, cuestión esta fácil de deducir a partir su definición. Vamos a ver cómo se utilizaría para trisectar un ángulo.

Consideremos que queremos trisectar el ángulo formado por la recta $r_{1}$, que para simplificar supondremos que es el eje OX, y la recta que pasa por el origen de coordenadas $r_{2}$. Se trata de buscar dos rectas $r_{3}$ y $r_{4}$ que pasen por el origen de coordenadas tales que $\angle\left(r_{3}, r_{4}\right)=\frac{1}{3} \angle\left(r_{1}, r_{2}\right)$. Sea $A$ el punto de intersección de la espiral con 
la recta $r_{2}$ y sean $P$ y $Q$ los puntos que dividen el segmento $O A$ en tres partes iguales. Estos puntos son fácilmente obtenibles utilizando el teorema de Tales. De este modo:

$$
d(O, A)=t ; d(O, P)=\frac{t}{3} ; d(O, Q)=\frac{2 t}{3}
$$

Consideramos ahora los puntos $N$ y $M$ donde los arcos de circunferencia de centro $O$ y radios $\frac{t}{3}$ y $\frac{2 t}{3}$ cortan a la espiral. Las coordenadas de estos puntos serán:

$$
N=\frac{t}{3}\left(\cos \left(\frac{t}{3}\right), \operatorname{sen}\left(\frac{t}{3}\right)\right) \text { y } M=\frac{2 t}{3}\left(\cos \left(\frac{2 t}{3}\right), \operatorname{sen}\left(\frac{2 t}{3}\right)\right)
$$

Si consideramos ahora las semirrectas $\mathrm{r} 3$ y r 4 de origen $O$ y que pasan respectivamente por $\mathrm{N}$ y $\mathrm{M}$ tendremos, como queríamos demostrar, que,

$$
\angle\left(r_{3}, r_{4}\right)=\frac{2 t}{3}-\frac{t}{3}=\frac{t}{3}=\frac{1}{3} \angle\left(r_{1}, r_{2}\right)
$$

Fuera de las Matemáticas, a esta espiral se le han encontrado una multitud de aplicaciones, como por ejemplo su uso para comprimir líquidos y gases, para la fabricación de los ya antiguos discos de vinilo o para medir el temblor humano que nos ayuda a diagnosticar posibles enfermedades neurológicas.

El problema que más ha contribuido en el desarrollo del Álgebra a lo largo de la historia ha sido sin duda la resolución de ecuaciones polinómicas mediante radicales. Ya en la antigua Babilonia conocían la forma de solucionar ecuaciones de segundo grado de una forma muy similar a como lo hacemos en la actualidad. Así nos lo muestran las tablillas encontradas de la época con ejemplos concretos que indican que conocían un método concreto para resolver este tipo de ecuaciones.

FIGURA 3

Método árabe de solucionar una ecuación de segundo grado

\begin{tabular}{|r|c|c|}
\hline$b / 4$ & $x$ & $b / 4$ \\
\hline$x$ & & \\
& & $x$ \\
& & \\
\hline$b / 4$ & & $b / 4$ \\
& & \\
\hline
\end{tabular}


En el siglo IX, Al-Khowarizmi obtuvo la fórmula de resolución de la ecuación de segundo grado $X^{2}+b X=c$ por métodos geométricos del siguiente modo. Si $x$ es una solución positiva de la ecuación (téngase en cuenta que en esa época no se consideraban soluciones negativas), construimos un cuadrado de lado $x$. Prolongamos los cuatro lados una longitud $\frac{b}{4}$ tal y como muestra la Figura 3. Si igualamos el área del cuadrado exterior con la suma de los cinco cuadrados y cuatro rectángulos interiores nos queda:

$$
\left(x+\frac{b}{2}\right)^{2}=x^{2}+4 \cdot\left(\frac{b}{4}\right)^{2}+4 \cdot \frac{b}{4} x=4 \cdot\left(\frac{b}{4}\right)^{2}+c
$$

Despejando $x$ obtenemos la fórmula que utilizamos en la actualidad con $a=1$ :

$$
x=\sqrt{4 \cdot\left(\frac{b}{4}\right)^{2}+c}-\frac{b}{2}=\sqrt{\frac{b^{2}}{4}+c}-\frac{b}{2}=\frac{-b+\sqrt{b^{2}+4 c}}{2}
$$

La resolución por radicales de la ecuación de grado tres y cuatro se resistió hasta el siglo Xvi. Fue el matemático italiano Tartaglia el que resolvió la ecuación cúbica por medio de radicales, mientras que otro matemático italiano, Ferrari, fue el que solucionó la ecuación polinómica de grado cuatro. Debido a la complejidad de estas expresiones, apenas se estudian y carecen de valor didáctico, al menos en la Enseñanza Secundaria. No obstante, desde un punto de vista matemático, lo importante es que se descubrió que existían estas expresiones mediante radicales que solucionaban estas ecuaciones.

El problema siguiente estaba claro: ¿se podría resolver por medio de radicales cualquier ecuación polinómica de grado cinco? Tres siglos más tarde Abel ${ }^{1}$ probó que en estos casos no era posible encontrar fórmulas generales de expresar las raíces de los polinomios mediante sumas, restas, multiplicaciones, divisiones y extracción de raíces a partir de los coeficientes de la ecuación.

Aún quedaba pendiente la cuestión de saber qué polinomios: $f(X)=X^{n}+a_{1} X+\mathrm{L}+a_{n} \in K(X)$, sí podían ser resueltos mediantes radicales. Este problema lo solucionó brillantemente el matemático francés Galois ${ }^{2}$. Probó la existencia de un cuerpo minimal $K(f)$ entre aquellos sobre los que $f$ se factoriza en producto de factores lineales, construyó el grupo $G(f)$ de los automorfismos de $K(f)$ que dejan fijos todos los elementos de $K$ y demostró que $f$ es resoluble por radicales si y solo si existe una familia finita de $\left\{G_{1}, \ldots, G_{\mathrm{n}}=G(f)\right\}$ de subgrupos de $G(f)$ tales que cada $G_{i}$ es subgrupo normal de $G_{i+1}$ y el cociente $G_{i+1} / G i$ es abeliano.

1. Puede consultarse en Abel, N. H. (1988). Oeuvres Complètes. Nueva York: Johnson Reprint Corp.

2. Sus hallazgos los publicó por primera vez el catedrático en Matemáticas Joseph Liouville en Mathématiques Pures et Appliquées en 1846. 
Todas estas ideas supusieron el desarrollo de lo que hoy se conoce como Álgebra Abstracta, que es la rama de las Matemáticas que estudia estructuras algebraicas tales como anillos, grupos, cuerpos, espacios vectoriales, etc. Sin duda, estas Matemáticas no estaban al alcance de los matemáticos del siglo XVI.

Otros dos problemas que han sido de una importancia vital en las Matemáticas han sido el de calcular la tangente a una curva en un punto de esta y el cálculo del área encerrada por una curva, ya que en ellos se encuentran el germen de Cálculo Diferencial e Integral.

Arquímedes en el siglo v a. C. ya empezó a tratar el segundo problema con los denominados métodos de exhaución y compresión con los que inicialmente intentó calcular la longitud de la circunferencia. La idea era muy sencilla. Dada una circunferencia se iba aproximando a su perímetro por medio de polígonos regulares inscritos (método de exhaución) y por medio de polígonos regulares circunscritos (método de compresión). De este modo y utilizando polígonos regulares de hasta 96 lados obtuvo una acotación de $\pi$ prodigiosa para la época en la que se encontraba:

$$
3,140845 \ldots<\pi<3,142857 \ldots
$$

El problema del cálculo de tangentes a curvas también interesó a los griegos. Euclides $^{3}$ definió en Los Elementos la tangente a una circunferencia como aquella recta que toca a la circunferencia pero no la corta. Se acabaron dando cuenta de que la tangente en un punto $P$ de una circunferencia es la recta perpendicular al radio de la circunferencia que pasa por $P$. Apolonio, en el siglo III a. C., encontró también métodos para calcular tangentes en cualquier cónica. Sin embargo, no supieron calcular tangentes en otros tipos de curvas como las algebraicas de grado tres o superior o en la espiral de Arquímedes.

Tras la decadencia de la escuela de Alejandría, el interés por este tipo de problemas decayó hasta la creación de la Geometría Analítica en el siglo XVII por parte de Fermat y Descartes. A partir de aquí, estos y otros matemáticos comenzaron a criticar los métodos griegos de cara a poder calcular tangentes y áreas utilizando para ello técnicas geométricas, algebraicas o incluso cinemáticas.

Destacaremos los métodos que propuso Fermat para el cálculo de tangentes y áreas encerradas por curvas. Para calcular la

\section{FIGURA 4}

Cálculo de la tangente de Fermat

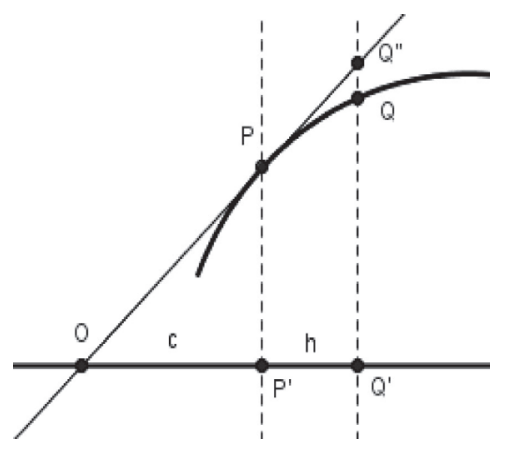

3. Se encuentra en la definición 2 del Libro III de Los Elementos. 
tangente en un punto $P(a, b)$ a una curva $\alpha$ de ecuación $y=f(x)$, lo que hace Fermat es considerar el punto $O$, que es el punto donde la recta tangente corta al eje $O X$, y el punto $P$, que será la proyección ortogonal del punto $P$ sobre el eje $O X$. Si $h$ es pequeño, el error que se puede cometer al suponer que el punto $Q(a+b$, $f(a+b))$ pertenece a la tangente es también pequeño.

Asumiendo ese error, podemos suponer entonces que los triángulos $O P P^{\prime}$ y OQQ' son semejantes. En ese caso, la pendiente de la recta tangente será:

$$
m=\frac{P P^{\prime}}{O P^{\prime}}=\frac{Q^{\prime \prime} Q^{\prime}}{O Q^{\prime}} \approx \frac{Q Q^{\prime}}{O Q^{\prime}}=\frac{f(a+h)}{c+h}=\frac{f(a+h)-b}{h}=\frac{f(a+h)-f(a)}{h}
$$

De este modo, cuando $h \rightarrow 0$, Fermat proponía como pendiente de la tangente a una curva lo que hoy en día se conoce como la derivada de la función en un punto.

Veamos a continuación la forma que tuvo de calcular el área encerrada por la curva $y=x^{n}$, el eje $O X$ y la recta $x=a$. Considera la partición formada por los puntos $a, a b, a b^{2}, \ldots$, donde $0<b<1$, y aproxima el área $A$ buscada por la suma de las áreas de los rectángulos superiores obtenidos tal y como muestra la figura. Observemos la semejanza de este método con el método de compresión utilizado 2000 años antes por Arquímedes.

\section{FIGURA 5}

Cálculo de la integral definida de Fermat

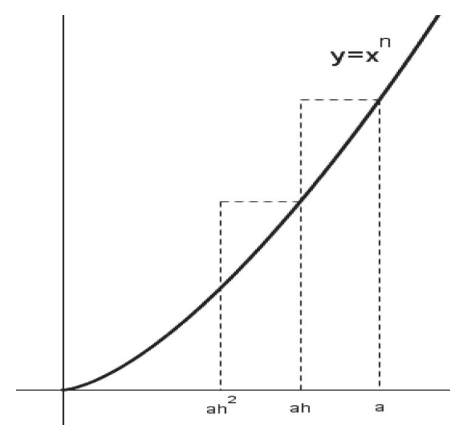

Tendremos por tanto que:

$$
A(h)=a^{n} \cdot(a-a h)+a^{n} h^{n} \cdot\left(a h-a h^{2}\right)+a^{n} h^{2 n} \cdot\left(a h^{2}-a h^{3}\right)+\cdots
$$

La suma anterior es la suma de los infinitos términos de una progresión geométrica de primer término $a-a b$ y razón $b^{1+n}$, con lo cual:

$$
A(h)=\frac{a^{n} \cdot(a-a h)}{1-h^{1+n}}=\frac{a^{n+1} \cdot(h-1)}{h^{1+n}-1}=\frac{a^{n+1}}{1+h+h^{2}+\cdots+h^{n}}
$$


Haciendo los rectángulos "infinitesimales", lo que equivale a que $b \rightarrow 1$, se obtiene finalmente que

$$
A=\lim _{h \rightarrow 1} A(h)=\frac{a^{n+1}}{n+1}
$$

A Fermat solo le faltó relacionar los dos problemas para ser considerado el inventor del cálculo. Este honor se lo llevaron Newton y Leibniz que sí fueron capaces de relacionar, de maneras diferentes, los problemas de las tangencias y el cálculo de aéreas encerradas por curvas apoyándose en una misma idea, despreciar los infinitésimos de mayor orden. Existe una gran polémica sobre quién de los dos fue el verdadero inventor del cálculo. Parece ser que Newton se adelantó en su descubrimiento, pero que Leibniz se adelantó en su publicación. Los métodos utilizados por Newton fueron más profundos, pero la notación de Leibniz era más adecuada de cara a seguir desarrollando esta rama de las Matemáticas.

Siguiendo con Fermat, no hay que olvidar el anteriormente mencionado «último teorema de Fermat.. Fermat en el margen de su copia del libro Arithmética de Diofanto escribió que había descubierto una demostración de este teorema, que él denominó admirable, pero que el margen de este libro era muy pequeño para desarrollarla. La demostración de este teorema ha sido buscada por los más grandes matemáticos durante más de tres siglos y gracias a ello se desarrolló la Teoría Algebraica de Números. En el año 1735, Euler demostró este teorema para el caso $n=3$, pero no fue hasta 1993 cuando Wiles demostró el teorema basándose para ello en formas modulares de curvas elípticas.

Fermat también es considerado como el propulsor, junto con el matemático francés Pascal, de la Teoría de Probabilidades. Todo se originó en 1654 por un intercambio de correspondencia entre ellos acerca de un problema que le había propuesto un jugador conocido como el caballero de Mere:

Dos jugadores apuestan 32 doblones de oro cada uno a un juego que consiste en obtener antes que el otro jugador tres veces un determinado número, tirando el dado una vez cada uno. Si el juego se interrumpe cuando un jugador ha obtenido dos veces la puntuación y el otro solo una, ¿̇cómo deben repartirse los doblones apostados?

El análisis de este y otros problemas y las correcciones que le hicieron al caballero de Mere, que consideraba equiprobables sucesos que no lo eran, supusieron el inicio de la formalización de la Teoría de Probabilidades.

Otro de los problemas relevantes en la historia de las Matemáticas fue el denominado "problema de los puentes de Königsberg». Este aparentemente senci-

\section{IMAGEN 1}

Problema de los puentes de Königsberg

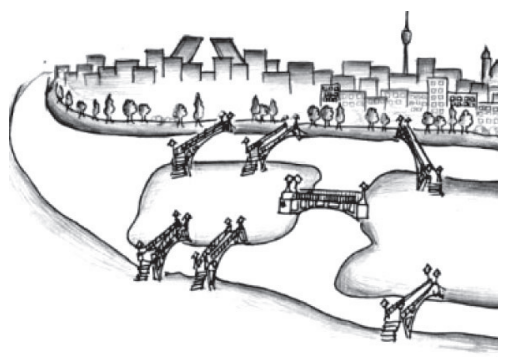

Nota. Fuente: http://www.expansion.com. 
llo juego planteado por Euler ${ }^{4}$ trata de decidir si se puede recorrer la ciudad de Königsberg, que es atravesada por el río Pregolya, pasando por sus siete puentes (ver Imagen 4) una y solo una vez, volviendo finalmente al punto inicial.

Este problema lo resolvió en 1736 el propio Euler viendo que dicho camino no existe ya que el grafo que genera el problema no es euleriano porque tiene vértices de grado impar. Todo esto dio origen a la denominada Teoría de Grafos, que es una rama de las Matemáticas que está en constante desarrollo y que ha permitido resolver otros muchos problemas como el problema de los cuatro colores con ayuda de los ordenadores.

En este punto hemos mostrado como algunas demostraciones y problemas han sido el motor del desarrollo de algunas ramas de las Matemáticas. Debido a la importancia histórica que han tenido no es descabellado preguntarse si deberían tener una importancia al menos similar en la didáctica de las Matemáticas en la Educación Secundaria.

\section{EXPERIENCIA EN EL AULA}

\subsection{Introducción}

Como hemos visto, las demostraciones y la resolución de problemas han jugado históricamente un papel fundamental en el desarrollo de las Matemáticas. Parece lógico por ello reservarle un espacio importante en la didáctica de las Matemáticas, y no sólo por la relevancia histórica que han tenido, sino porque es una herramienta fundamental en el aula de cara a mejorar el razonamiento lógico del alumno.

Existe unanimidad entre los investigadores en considerar los problemas matemáticos como una herramienta indispensable en la didáctica de las Matemáticas. Este consenso no es tal cuando hablamos de la utilización didáctica de la demostración en aula. Autores como por ejemplo Polya o Hanna apoyaban el uso de las demostraciones. Polya (1945) hizo especial hincapié en el uso de las demostraciones geométricas con el objetivo de que alumno no pierdiera la ocasión de saber lo que es un razonamiento lógico riguroso, mientras que Hanna (1995) se centró más en la forma de presentar la demostración en aula, afirmando que no debe hacerse como un puro ritual, sino como un procedimiento con "razón de ser" dentro del aprendizaje.

Sin embargo, otros autores de gran relevancia como Lakatos o Kline desaconsejaron su utilización. Lakatos (1976) sostenía la idea de que la utilización didáctica de la demostración dificultaba la construcción del conocimiento matemático, sobre

4. Publicado en Euler, L. (1736). Solutio problematis ad geometriam situs pertinentis (Reimpreso en Opera Omnia Series Prima 1976) [En red]. Disponible en: http://math.darmouth.edu/ euler/docs/ originals/E053.pdf, 128-140 (versión original en latín). 
todo en los alumnos que no estudiaban para ser matemáticos y que en su gran mayoría no estaban preparados para apreciar la belleza de las demostraciones y motivarse para llevarlas a cabo. Kline (1981) planteó una serie de argumentos para defender su postura contraria al uso didáctico de las demostraciones:

a) Muchos matemáticos han descubierto teoremas de una gran importancia que luego no han sabido demostrar.

b) Al dar demasiada importancia al rigor, se pueden alejar las Matemáticas de los estudiantes al parecer que sus resultados provienen de personas con un alto nivel intelectual que razonan directamente con teoremas y axiomas.

c) No son procedimientos útiles para solucionar problemas cotidianos.

d) Los planteamientos deductivos pueden resultar motivadores para cierto perfil del profesorado, pero son anestésicos para la gran generalidad del alumnado.

En este artículo, vamos a describir una experiencia práctica mediante la cual se muestra como con un uso apropiado de la demostración los alumnos adquieren una serie de destrezas que les llevan a mejorar sus procedimientos en la resolución de problemas.

\subsection{Planteamiento del problema}

El problema de nuestra experiencia consistió en comprobar si, aplicando una didáctica de las Matemáticas para un grupo de $3 .^{\circ}$ ESO en donde se le dé un papel relevante a las demostraciones matemáticas, se mejoran las habilidades de los alumnos a la hora de resolver problemas matemáticos. Para ello compararemos los resultados obtenidos por este grupo en tres pruebas de resolución de problemas (una por trimestre) con los obtenidos por otro grupo del mismo nivel en el que se ha llevado a cabo una metodología más tradicional basada en explicaciones, realización de ejercicios prácticos y actividades con nuevas tecnologías.

\subsection{Proceso de la investigación}

La investigación se realizó en el instituto público Rafael Alberti de Coslada (Madrid). Utilizamos dos grupos de $3 .^{\circ}$ EsO, los dos con 21 alumnos, considerando uno como grupo control y el otro como experimental.

En el grupo control aplicamos una metodología más tradicional basada en explicaciones, realización de ejercicios, actividades TIC, etc. En el grupo experimental utilizamos una metodología en donde dimos una especial relevancia a argumentar y a justificar resultados. A lo largo del curso realizamos las siguientes demostraciones: 
1. Demostrar la irracionalidad de $\sqrt{2}$ por el método de reducción al absurdo.

2. Demostrar de forma directa las fórmulas de la suma de un número finito de términos de una progresión aritmética y de la suma de un número finito e infinito (cuando $|\mathrm{r}|<1$ ) de una progresión geométrica.

3. Demostrar las fórmulas de las identidades notables de un modo algebraico directo y de un modo geométrico.

4. Demostrar, utilizando las identidades notables, la fórmula de resolución de la ecuación de segundo grado.

5. Demostrar, utilizando para ello el programa Geogebra, la localización del vértice de una parábola mediante traslaciones.

6. Demostrar distintas propiedades geométricas sencillas como que la suma de los ángulos interiores de un triángulo suman $180^{\circ}$ o que las tres mediatrices de un triángulo se cruzan en un punto. Para ello, aparte de una demostración rigurosa, justificamos que esto realmente ocurre en un gran número de casos utilizando el programa Geogebra. Estrictamente hablando, trabajar con Geogebra estos hechos no supone realizar una demostración, pero sí hace entender al alumno lo que vamos a demostrar y le hace comprender de antemano que lo que demostraremos es cierto.

7. Demostrar de distintas formas geométricas el teorema de Pitágoras. También utilizamos Geogebra para comprobar y justificar este resultado.

Posteriormente analizaremos e interpretaremos los resultados obtenidos desde un punto de vista evolutivo, comprobando si existían diferencias significativas entre los grupos inicialmente y si se han producido diferencias significativas en las pruebas de resolución de problemas que realizamos a lo largo del curso.

\subsection{Hipótesis planteadas}

En nuestra experiencia vamos a utilizar cuatro momentos: inicial, primer trimestre, segundo trimestre y tercer trimestre. En el momento inicial vamos a comprobar que entre los dos grupos no existían diferencias significativas en torno a dos variables, las calificaciones en la asignatura de Matemáticas el curso anterior y las calificaciones obtenidas por los alumnos en la preceptiva evaluación inicial. Por tanto, las hipótesis nulas serán que no hay diferencias significativas entre los dos grupos en torno a estas variables, mientras que las hipótesis alternativas serán que sí existen estas diferencias significativas.

En los respectivos trimestres plantearemos la misma situación en torno a los resultados de las pruebas de resolución de problemas que les propusimos a los alumnos. De nuevo, las hipótesis nulas serán que no hay diferencias significativas en las medias entre los grupos en los resultados de estas pruebas, mientras que las hipótesis alternativas serán que sí existen estas diferencias significativas. 
ENRIQUE SÁNCHEZ FREIRE Y JUAN ANTONIO GIL PASCUAL

LA DEMOSTRACIÓN EN LAS MATEMÁTICAS..

\section{IMAGEN 2}

Apletts de Geogebra utilizados para demostrar la localización del vértice de una parábola
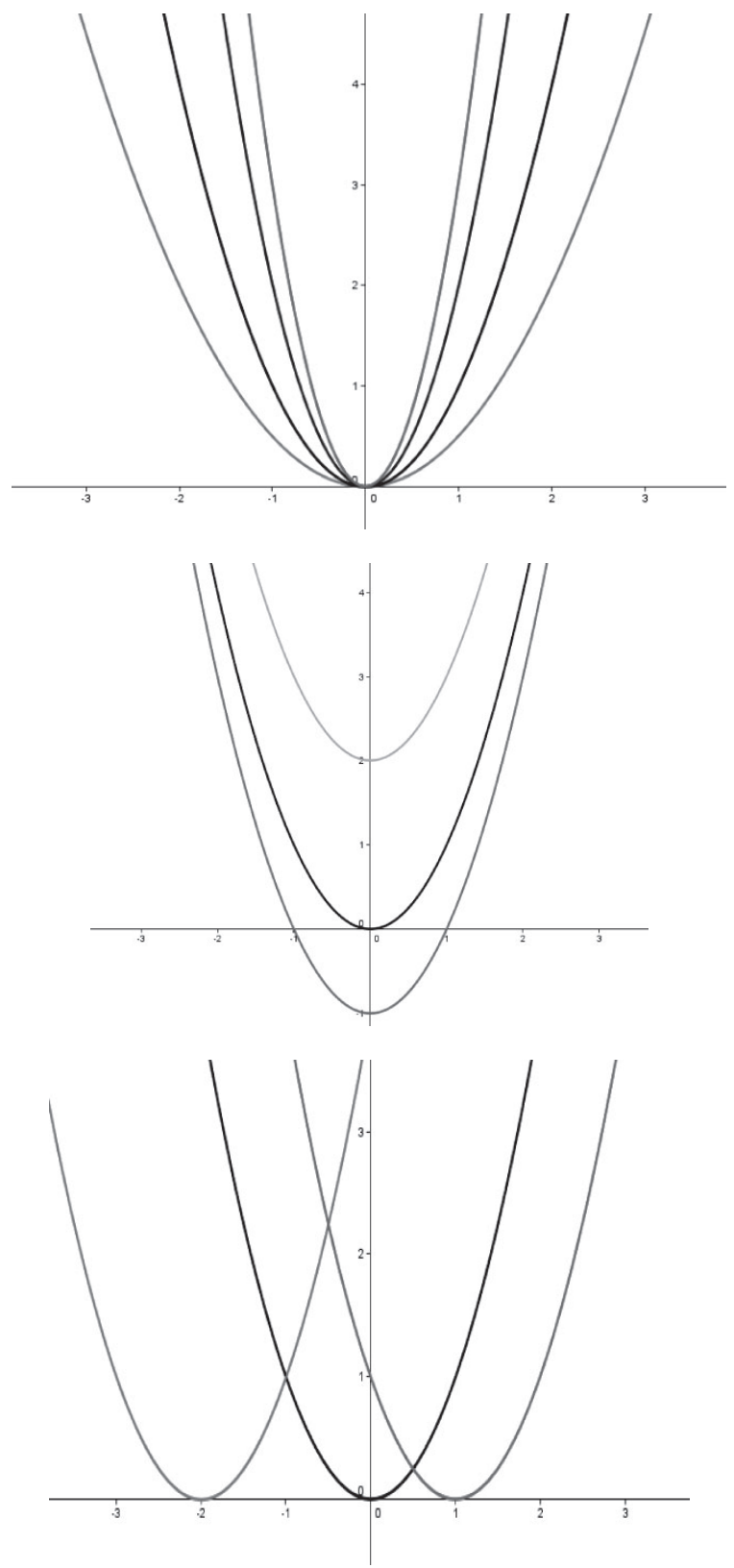

(c) Ediciones Universidad de Salamanca

Enseñanza \& Teaching, 33, 1-2015, pp. 163-192 


\section{IMAGEN 3}

Aplett de Geogebra utilizado para demostrar que la suma de los ángulos interiores de un triángulo es $180^{\circ}$

C

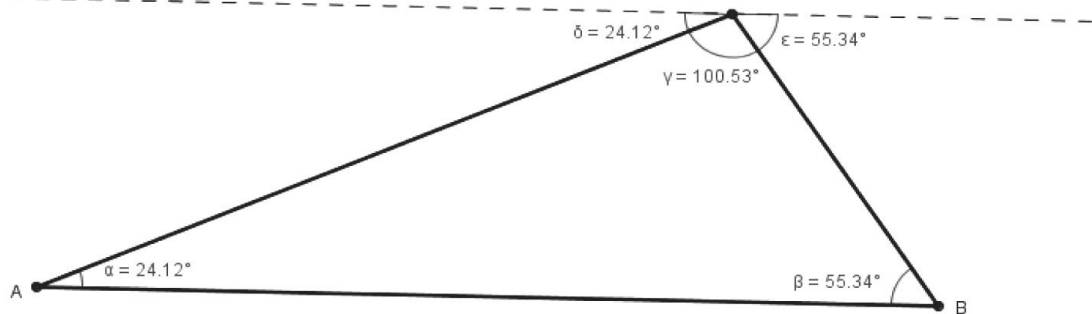

IMAGEN 4

Aplett de Geogebra utilizado para demostrar que las tres mediatrices de cualquier triángulo se cortan en un punto

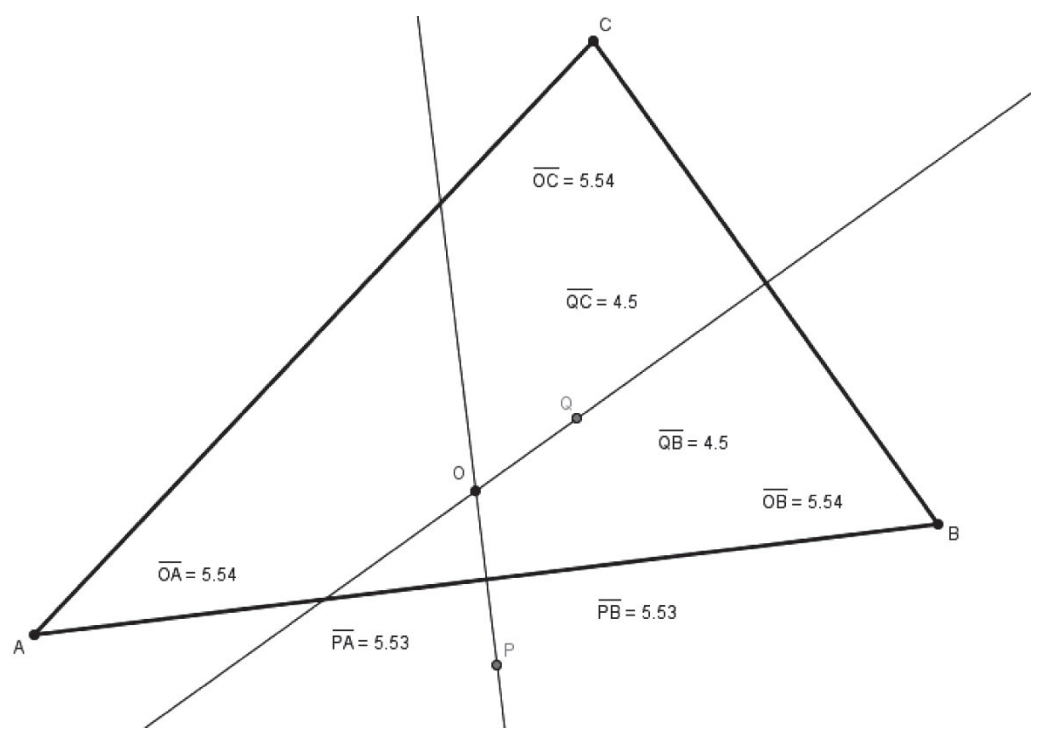




\section{IMAGEN 5}

Aplett de Geogebra utilizado para justificar el teorema de Pitágoras

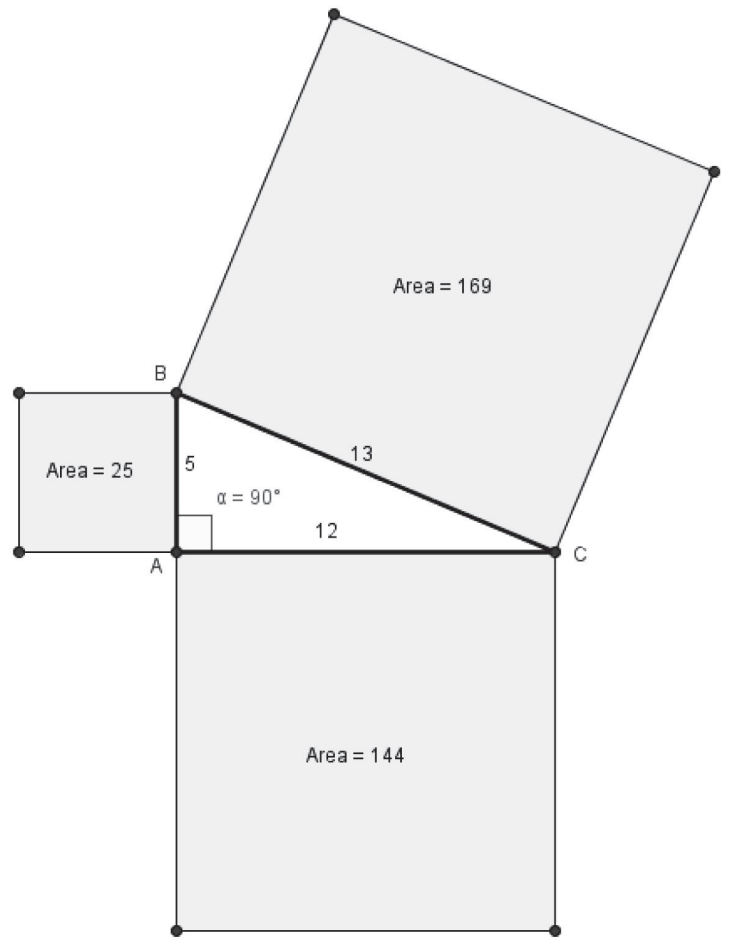

\subsection{Técnicas e instrumentos de recogida de la información}

Los instrumentos que utilizamos en nuestra experiencia fueron:

a) Una prueba de evaluación inicial con contenidos tratados el curso anterior que se realizó el 26 de septiembre de 2011.

b) Tres pruebas de resolución de problemas que se realizaron respectivamente los días 21 de noviembre de 2011, el 20 de febrero de 2012 y el 28 de mayo de 2012. Estas prueba constaron de ocho problemas e intentamos que en cada una estuvieran los distintos tipos de problemas que pueden resolver alumnos de estas edades: Geometría (descomposición adecuada de una figura, petición de un elemento concreto, etc.), Aritmética (buscar operaciones adecuadas para llegar a la solución, técnicas de conteo, etc.) y Lógica (buscar un razonamiento que resuelva el problema). Para comprobar que eran adecuados para el nivel de los alumnos 
pedimos mediante una encuesta la opinión de 27 expertos en la materia sobre cada uno de los problemas propuestos. Estas pruebas se incluirán como anexo al artículo.

\subsection{Diseño de la experiencia}

Utilizaremos un enfoque experimental, ya que a partir de una experimentación buscaremos una ley general estableciendo relaciones causales entre variables utilizando técnicas estadísticas. Debido a que trabajaremos con muestras y no tendremos por tanto el control absoluto de las variables, el diseño de la investigación será del tipo cuasi-experimental.

\subsection{Análisis estadístico a realizar}

En este artículo nos basaremos en un estudio estadístico inferencial. Comprobaremos mediante la técnica del análisis de la varianza (ANOVA) si se han producido diferencias significativas en las variables que mencionamos anteriormente. Para los cálculos utilizamos el programa de software libre $\mathrm{R}$ y como es habitual en Estadística rechazaremos la hipótesis de igualdad de medias cuando el nivel crítico asociado al estadístico sea menor que 0,05 , no rechazando la igualdad de medias en caso contrario.

\subsection{Resultados}

En el momento inicial teníamos que comprobar si existían diferencias significativas entre los dos grupos en torno a dos variables: las calificaciones en la asignatura de Matemáticas el curso anterior y los resultados en la prueba de evaluación inicial. Los resultados de los análisis de la varianza se muestran en las siguientes tablas, en donde los datos ausentes que aparecen en la evaluación inicial se debieron a alumnos que se incorporaron con posterioridad al centro:

TABLA 1

Análisis de la varianza de los resultados del curso anterior en Matemáticas en función de los grupos.

\begin{tabular}{|c|c|c|c|c|c|}
\hline & $\begin{array}{c}\text { GRADOS DE } \\
\text { LIBERTAD }\end{array}$ & $\begin{array}{c}\text { SUMA DE } \\
\text { CUADRADOS }\end{array}$ & $\begin{array}{c}\text { MEDIA } \\
\text { CUADRÁTICA }\end{array}$ & VALOR DE F & $\mathrm{P}(>\mathrm{F})$ \\
\hline Grupo & 1 & 0,38 & 0,381 & 0,059 & 0,809 \\
\hline Residuales & 40 & 258,10 & 6,452 & & \\
\hline
\end{tabular}


Su correspondiente tabla de descriptivos fue la siguiente:

TABLA 2

Descriptivos de los resultados del curso anterior en Matemáticas

\begin{tabular}{|c|c|c|c|c|}
\hline & MEDIA & DESVIACIÓN TÍPICA & NÚMERO DE DATOS & DATOS AUSENTES \\
\hline Experimental & 5,619048 & 2,889225 & 21 & 0 \\
\hline Control & 5,428571 & 2,134747 & 21 & 0 \\
\hline
\end{tabular}

TABLA 3

Análisis de la varianza de los resultados de la evaluación inicial en función de los grupos

\begin{tabular}{|c|c|c|c|c|c|}
\hline & $\begin{array}{c}\text { GRADOS DE } \\
\text { LIBERTAD }\end{array}$ & $\begin{array}{c}\text { SUMA DE } \\
\text { CUADRADOS }\end{array}$ & $\begin{array}{c}\text { MEDIA } \\
\text { CUADRÁTICA }\end{array}$ & VALOR DE F & $\mathrm{P}(>\mathrm{F})$ \\
\hline Grupo & 1 & 0,01 & 0,005 & 0 & 0,967 \\
\hline Residuales & 38 & 121,21 & 3,190 & & \\
\hline
\end{tabular}

Su correspondiente tabla de descriptivos fue la siguiente:

TABLA 4

Descriptivos de la evaluación inicial

\begin{tabular}{|c|c|c|c|c|}
\hline & MEDIA & DESVIACIÓN TÍPICA & NÚMERO DE DATOS & DATOS AUSENTES \\
\hline Experimental & 2,671053 & 1,806033 & 19 & 2 \\
\hline Control & 2,647619 & 1,767801 & 21 & 0 \\
\hline
\end{tabular}

Si observamos los valores críticos obtenidos, ninguno de los dos son menores que 0,05 , con lo cual podemos afirmar que no existian diferencias significativas en las medias entre los dos grupos inicialmente en estas dos variables, lo cual es satisfactorio para la experiencia ya que los resultados indican un equilibrio inicial entre los grupos.

Ahora vamos a comparar estos datos con los resultados obtenidos por los alumnos en las respectivas pruebas de resolución de problemas de cada uno de los trimestres. Podremos observar que hay datos ausentes que se deben a bajas que se producen a lo largo del curso por diversas causas. Los resultados los podemos observar en las siguientes tablas: 
TABLA 5

Análisis de la varianza de los resultados de la primera prueba de problemas en función de los grupos

\begin{tabular}{|c|c|c|c|c|c|}
\hline & $\begin{array}{c}\text { GRADOS DE } \\
\text { LIBERTAD }\end{array}$ & $\begin{array}{c}\text { SUMA DE } \\
\text { CUADRADOS }\end{array}$ & $\begin{array}{c}\text { MEDIA } \\
\text { CUADRÁTICA }\end{array}$ & VALOR DE F & $\mathrm{P}(>\mathrm{F})$ \\
\hline Grupo & 1 & 5,87 & 5,869 & 1,098 & 0,301 \\
\hline Residuales & 40 & 213,72 & 5,343 & & \\
\hline
\end{tabular}

Su correspondiente tabla de descriptivos fue la siguiente:

TABLA 6

Descriptivos de los resultados de la primera prueba de problemas

\begin{tabular}{|c|c|c|c|c|}
\hline & MEDIA & DESVIACIÓN TÍPICA & NÚMERO DE DATOS & DATOS AUSENTES \\
\hline Experimental & 3,652381 & 2,721419 & 21 & 0 \\
\hline Control & 2,904762 & 1,811001 & 21 & 0 \\
\hline
\end{tabular}

\section{TABLA 7}

Análisis de la varianza de los resultados de la segunda prueba de problemas en función de los grupos

\begin{tabular}{|c|c|c|c|c|c|}
\hline & $\begin{array}{c}\text { GRADOS DE } \\
\text { LIBERTAD }\end{array}$ & $\begin{array}{c}\text { SUMA DE } \\
\text { CUADRADOS }\end{array}$ & $\begin{array}{c}\text { MEDIA } \\
\text { CUADRÁTICA }\end{array}$ & VALOR DE F & $\mathrm{P}(>\mathrm{F})$ \\
\hline Grupo & 1 & 18,41 & 18,407 & 6,737 & 0,0132 \\
\hline Residuales & 39 & 106,55 & 2,732 & & \\
\hline
\end{tabular}

Nota: Valores significativos $p<.05$; valores muy significativos $p<.01$; valores altamente significativos $p<.001$.

Su correspondiente tabla de descriptivos es la siguiente:

TABLA 8

Descriptivos de los resultados de la segunda prueba de problemas

\begin{tabular}{|c|c|c|c|c|}
\hline & MEDIA & DESVIACIÓN TÍPICA & NÚMERO DE DATOS & DATOS AUSENTES \\
\hline Experimental & 3,250000 & 2,114735 & 20 & 1 \\
\hline Control & 1,909524 & 1,038824 & 21 & 0 \\
\hline
\end{tabular}


TABLA 9

Análisis de la varianza de los resultados de la tercera prueba de problemas en función de los grupos

\begin{tabular}{|c|c|c|c|c|c|}
\hline & $\begin{array}{c}\text { GRADOS DE } \\
\text { LIBERTAD }\end{array}$ & $\begin{array}{c}\text { SUMA DE } \\
\text { CUADRADOS }\end{array}$ & $\begin{array}{c}\text { MEDIA } \\
\text { CUADRÁTICA }\end{array}$ & VALOR DE F & $\mathrm{P}(>\mathrm{F})$ \\
\hline Grupo & 1 & 27,02 & 27,019 & 7,268 & 0,0104 \\
\hline Residuales & 38 & 141,27 & 3,718 & & \\
\hline
\end{tabular}

Nota: Valores significativos $p<.05$; valores muy significativos $p<.01$; valores altamente significativos $p<.001$.

Su correspondiente tabla de descriptivos es la siguiente:

TABLA 10

Descriptivos de los resultados de la tercera prueba de problemas

\begin{tabular}{|c|c|c|c|c|}
\hline & MEDIA & DESVIACIÓN TÍPICA & NÚMERO DE DATOS & DATOS AUSENTES \\
\hline Experimental & 3,12875 & 2,421916 & 20 & 1 \\
\hline Control & 1,48500 & 1,252928 & 20 & 1 \\
\hline
\end{tabular}

Si observamos los tres valores críticos obtenidos en la primera prueba no se obtuvieron diferencias significativas en las medias entre los grupos, pero que tanto en la segunda como en la tercera prueba si que se obtuvieron diferencias significativas a favor del grupo experimental, alcanzándose valores próximos a considerar esas diferencias como muy significativas.

\section{CONCLUSIONES Y LIMITACIONES DE LA INVESTIGACIÓN}

Hemos podido comprobar que progresivamente los alumnos del grupo experimental han ido obteniendo mejores calificaciones en las pruebas de resolución de problemas en relación a las que obtuvieron los alumnos del grupo control, confirmando de este modo las hipótesis planteadas.

Resultaría un error considerar que las diferencias que se han obtenido en los resultados se deben en exclusiva a las diferencias metodológicas que hemos empleado con sendos grupos. En la asignatura de Matemáticas y en el resto de las asignaturas pudimos observar como el grupo experimental fue obteniendo a lo largo del curso mejores resultados que el grupo control, aunque es cierto que las diferencias más significativas se presentaron tanto en la asignatura de Matemáticas, como en estas pruebas de resolución de problemas que les propusimos.

También debemos ser conscientes de que esta es una experiencia puntual, con dos grupos concretos. Se podría extender a otros cursos, a centros de otra tipología (concertados o privados), realizar la misma experiencia pero con una temporalización mayor, etc. Todo esto con el objetivo de comprobar si los resultados siguen la tendencia que aquí han presentado. 
Esta experiencia ha reforzado nuestra opinión inicial sobre el uso de las demostraciones. Creemos que la forma que hemos utilizado en esta experiencia para introducir la demostración a nuestros alumnos no es la ideal. Aunque la hemos introducido de forma progresiva a lo largo del curso, creemos que este trabajo se debe iniciar antes, incluso en la Educación Primaria realizando pequeñas pruebas y conjeturas. Se trataría de ir acostumbrando a los alumnos a los procesos de justificación acordes al curso en el que se encuentren y al nivel propio de los alumnos, e inculcarles una actitud crítica en los resultados que van apareciendo.

En resumen, nuestras propuestas van en dos líneas concretas:

a) $\mathrm{Al}$ poder legislativo de este país, le proponemos que introduzca estos procesos en el currículo de una forma más explícita, indicando qué tipo de razonamientos y demostraciones se pueden proponer en cada curso, para que progresivamente vayamos alcanzando unas Matemáticas más razonadas y rigurosas a lo largo de la Educación Secundaria.

b) Proponemos que haya una formación adecuada del profesorado de Educación Primaria y Secundaria sobre cómo, cuándo y qué demostrar en cada curso, con el objetivo de dejar de lado esas Matemáticas tan algorítmicas que en ocasiones generan nuestros alumnos.

\section{REFERENCIAS BIBLIOGRÁFICAS}

Arsac, G. (1987). El origen de la demostración: ensayo de epistemología didáctica. Recherches en didactique des mathematiques, 8 (3), 267-312. (Martín Acosta, trad.). [En red] Disponible en: http://www-didactique.imag.fr/preuve /Resumes/Arsac/ Arsac02.pdf.

Balacheff, N. (2000). Procesos de prueba en los alumnos de matemáticas. (Pedro Gómez, trad.) Bogotá: Universidad de los Andes.

Bombal, F. (2010). Rigor y demostración en Matemáticas. Revista de la Real Academia de Ciencias Exactas, Física y Naturales (104), 1, 61-79.

Chaitin, G. J. (2003). Ordenadores, paradojas y fundamentos de las matemáticas. Investigación y Ciencia, 322, 28-35.

Gamboa, J. M. y Rodríguez, B. (2008). Desarrollo del temario de las oposiciones de secundaria (vols. 1 y 2). Madrid: Sanz y Torres.

Hanna, G. (1995). Challenges to the importance of proof. For the learning mathematics, (15), 3, 42-49.

Hanna, G. (2007). The ongoing value of proof. En Theorems in schools: from history, epistemology and cognition to classroom practice (pp. 3-18). Rotterdam: Sense Publishers.

Kline, M. (1981). El fracaso de la matemática moderna. Madrid: Siglo Veintiuno editores.

Lakatos, I. (1976). Proofs and refutations: The logic of mathematical discovery. Cambridge: Cambridge University Press.

http://dx.doi.org/10.1017/CBO9781139171472

Polya, G. (1945). How to solve it. Nueva Jersey: Princeton University Press. 
ANEXO: PRUEBAS DE PROBLEMAS PROPUESTAS

Los problemas de la primera prueba y sus respectivas soluciones que se les pasaron a los alumnos el 21 de noviembre de 2011 fueron:

1. ¿Cuántos números capicúas hay de dos cifras? ¿Y de tres cifras? ¿Y de cuatro cifras? Nota: los números capicúas son los que se leen igual de izquierda a derecha, que de derecha a izquierda.

Solución: Se trataba de un simple problema de conteo:

a) Números capicúas de dos cifras: son de la forma $a a$, luego hay 9 números capicúas de dos cifras.

b) Números capicúas de tres cifras: son de la forma $a b a$. Para $a=1$, habrá 10 números, para $a=2$ otros 10, etc. Es decir, hay un total de 90 números capicúas de tres cifras.

c) Números capicúas de cuatro cifras: son de la forma abba. Para $a=1$, habrá 10 números, para $a=2$ otros 10, etc. Es decir, hay un total de $\mathbf{9 0}$ números capicúas de cuatro cifras.

2. Un joyero consigue una rebaja de $150 €$ en la compra de 20 relojes iguales, cuyo precio según el catálogo es de 65 € la unidad. ¿A cuánto debe vender cada uno para obtener un beneficio total de $400 €$ ?

Solución: Este problema se puede hacer aritméticamente de varias formas. Por ejemplo, se sabe que, según catálogo, el joyero debió pagar 65 × $20=$ $1.300 €$. Como obtuvo una rebaja de $150 €$, realmente pagó $1.150 €$ por 20 relojes. Como quiere obtener $400 €$ de beneficio, con la venta debe obtener 1.150 $+400=1.550 €$, con lo que cada reloj debe venderlo a 1.550: $20=\mathbf{7 7 , 5} €$.

3. A una excursión Silvia y Rosa se llevaron respectivamente 5 y 4 pastelitos. Laura no se llevó ninguno, pero sus amigas decidieron compartir sus pastelitos con ella a partes iguales. En compensación, Laura les dio los 2,40€ que llevaba encima. ¿Cómo deberían repartirse ese dinero Silvia y Rosa?

Solución: Este problema es de razonamiento lógico. Como en total había 9 pastelitos, cada una tocó a 3 pastelitos. Ahora bien, Silvia, como llevaba 5, se puede decir que le dio 2 a Laura, y Rosa, como llevaba 4, se puede decir que le dio 1. Por lo tanto, los 2,40€ que pagó Laura por 3 pastelitos se deben repartir a razón de $\mathbf{1 , 6 0} €$ para Silvia y $0,80 €$ a Rosa.

4. En un restaurante te ofrecen el menú del día por $10 €$. El menú consiste en un primer plato a elegir entre 5 posibilidades, un segundo plato a elegir entre 4 posibilidades y un postre a elegir entre 4 posibilidades. También incluye el pan y la bebida, en la que puedes elegir agua, vino o un refresco. ¿Cuántos menús diferentes se pueden formar?

Solución: Este es un simple problema de conteo en donde las posibilidades totales serán la multiplicación del número de opciones de cada apartado, es decir, $5 \times 4 \times 4 \times 3=\mathbf{2 4 0}$ menús diferentes se pueden formar.

5. Deduce cuál es el mayor número entero positivo $n$ para el que se verifica que $n^{2000}<5^{3000}$. 
Solución: Es un problema que se resuelve con las propiedades de las potencias:

$$
n^{2000}<5^{3000} \Leftrightarrow\left(n^{2}\right)^{1000}<\left(5^{3}\right)^{1000} \Leftrightarrow n^{2}<125
$$

Claramente, el mayor número entero que cumple esa propiedad es $\mathbf{1 1}$.

6. En los tres primeros exámenes de una evaluación un alumno ha obtenido unas notas de 5,5 en el primero, 5,1 en el segundo y 3,2 en el tercero. ¿Qué nota debe sacar en el cuarto y último examen para que la media le dé 5? ¿Y para obtener una nota media de 6 ?

Solución: Para obtener una media de 5 con 4 exámenes, el alumno debe acumular como mínimo 5 × $4=20$ puntos. Como lleva acumulados en los tres primeros exámenes 13,8 puntos le harán falta $\mathbf{6 , 2}$ puntos en el último examen para obtener una media de 5 . Para obtener una nota media de 6 debería acumular 6 × $4=24$ puntos. Como lleva 13,8 puntos será imposible que alcance la media de 6 puntos.

7. A 50 alumnos de $3 .^{\circ}$ ESO se les ha preguntado si les gusta jugar al fútbol y al baloncesto. En los resultados aparece que a 35 personas les gusta el fútbol, a 22 personas les gusta el baloncesto y a 15 alumnos les gustan ambos deportes. ¿A cuántos alumnos no les gustan ni el fútbol, ni el baloncesto?

Solución: Con los datos que tenemos, sabemos que hay $35-15=20$ alumnos a los que solo les gusta el fútbol, $22-15=7$ alumnos a los que solo les gusta el baloncesto y 15 alumnos a los que les gustan los dos deportes. Por tanto, habrá $50-20-7-15=\mathbf{8}$ alumnos a los que no les gusta ninguno de los dos deportes.

8. En un decágono regular hemos unido mediante segmentos cada vértice con los restantes no consecutivos tal y como se observa en la figura. ¿Cuántos segmentos (interiores) hemos trazado en total?

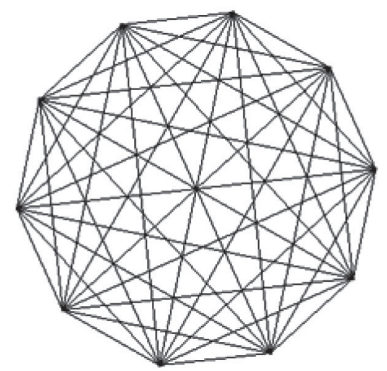

Solución: De cada uno de los 10 vértices salen 7 segmentos interiores. En principio podría parecer que entonces hay un total de 70 segmentos interiores, pero hay que darse cuenta de que cada segmento, al pertenecer a dos vértices, lo hemos contado dos veces, con lo que en total hay $70: 2=\mathbf{3 5}$ segmentos interiores. 
Los problemas de la segunda prueba, que se realizó el 20 de febrero de 2012, y sus respectivas soluciones fueron:

1. Si escribes todos los números naturales del 1 al 200 (ambos inclusive), ¿cuántas veces tienes que escribir la cifra "1"?

Solución: Veamos primero cuántas veces se escribe del 1 al 99. En las cifras de las unidades el 1 aparece 10 veces, mientras que en las cifras de las decenas aparece otras 10. En total 20 veces. Del 100 al 199, el 1 aparece 10 veces en las unidades, otras 10 veces en las decenas y 100 en las centenas, lo que hace un total de 120 veces. Luego, para escribir los números de 1 al 200, la cifra 1 la tendremos que utilizar $20+120=\mathbf{1 4 0}$ veces.

2. El presidente del C.F. Coslada ha fichado al goleador japonés Yono Fallouna por un año. Le va a pagar $800.000 €$ y un chalet en la mejor zona de Coslada. Al cabo de cuatro meses y ver que no mete ni un gol se le despide del equipo. Entre el presidente y el jugador acuerdan que solo va a recibir como pago el chalet y $100.000 €$. ¿En cuánto está valorado el chalet?

Solución: El sueldo anual del jugador es de $800.000 €+$ un chalet. Los cuatro meses trabajados equivalen a un tercio de año, con lo que por los dos tercios de año no trabajados ha dejado de percibir $700.000 €$. Luego, el sueldo del jugador por un tercio de año es de $350.000 €$. Si por el tercio de año trabajado le han pagado $100.000 €$ más el chalet, esto quiere decir que el chalet se ha

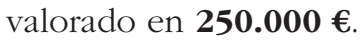

3. En una habitación hay taburetes de tres patas y sillas de cuatro patas. Cuando hay una persona sentada en cada uno de ellos, el número total de patas y piernas es 27. ¿Cuántos asientos hay?

Solución: Es un problema que se puede hacer por tanteo. Cada silla contaría como $6(4+2)$ patas y cada taburete como $5(3+2)$ patas.

- Si hubiera 1 silla, faltarían 21 patas, que no es múltiplo de 5.

- $\quad$ Si hubiera 2 sillas, faltarían 15 patas, lo que equivale a 3 taburetes.

- $\quad$ Si hubiera 3 sillas, faltarían 9 patas, que no es múltiplo de 5.

- Si hubiera 4 sillas, faltarían 3 patas, lo que es imposible.

Con lo cual, la única solución posible es que haya 2 sillas y 3 taburetes.

4. En un instituto hay tres grupos de $3 .^{\circ}$ ESO. El profesor de Educación Física manda hacer grupos de 6 alumnos cada uno. En el grupo A han quedado sin pertenecer a ningún equipo 4 alumnos, en el grupo B han quedado 5 alumnos y en el grupo $\mathrm{C}$ han quedado 3 alumnos. Si en otra actividad, el profesor junta a los tres grupos y vuelve a mandar que se emparejen en grupos de 6 , ¿cuántos alumnos quedarán sin equipo?

Solución: Si junta los grupos, podemos suponer que los grupos que se han hecho en cada clase se pueden mantener. Con lo que los $4+5+3=12$ alumnos que estaban sin equipo, se pueden hacer dos grupos más y no quedarian alumnos sin equipo. 
5. En la secuencia de letras ABCDEFABCDEFABCDEF... ¿Qué letra ocupa la posición 2012?

Solución: Fijémonos que es una secuencia que se repite periódicamente en grupos de 6, con lo cual tenemos que ver la posición que nos dan, que es 2012, donde caerá. Si dividimos 2012 entre 6, el cociente será 335 y el resto, que es lo importante, es 2 . Si vemos la secuencia, todos los números que tienen resto 2 al dividirlos entre 6, como son 2, 8, 14, 20, etc., coinciden en la letra, que es la B, con lo cual, en la posición 2012 irá una B.

6. Una liebre aventaja en 12 de sus saltos al galgo que la persigue. Dos saltos del galgo equivalen, en longitud, a tres de la liebre. Si el galgo tarda en dar tres saltos lo mismo que la liebre en dar cuatro, ¿cuántos saltos dará la liebre antes de ser alcanzada?

Solución: En tiempo, cada $3 \mathrm{G}=4 \mathrm{~L}$, o lo que equivale a que $6 \mathrm{G}=8 \mathrm{~L}$. Ahora bien, como en longitud $3 \mathrm{~L}=2 \mathrm{G}$, podemos asegurar que en el mismo tiempo que la liebre da 8 saltos, el galgo da 6 de los suyos que equivalen a 9 de la liebre. Es decir, que la liebre cada 8 saltos pierde un salto de los suyos de ventaja respecto al galgo. Como le sacaba 12 de sus saltos de ventaja, la liebre será alcanzada por el galgo cuando esta dé 12 × $8=96$ saltos.

7. Entre Javier y Lorenzo tienen 16 canicas. Entre Javier y David tienen 13 canicas. Entre David y Lorenzo tienen 17 canicas. ¿Cuántas canicas tiene cada uno de los tres?

Solución: Expresemos las tres informaciones de la siguiente manera:

$$
\mathrm{J}+\mathrm{L}=16 ; \mathrm{J}+\mathrm{D}=13 ; \mathrm{D}+\mathrm{L}=17
$$

Si sumamos las tres expresiones nos queda: $2 \mathrm{~J}+2 \mathrm{~L}+2 \mathrm{D}=46$. Con lo cual, entre los tres tienen 23 canicas. Ahora ya es fácil deducir que David tiene 7 canicas, Luis tiene 10 canicas y Javier tiene 6 canicas.

8. A las tres en punto, las agujas de un reloj forman un ángulo de 90'. ¿Qué ángulo (agudo) formarán esas agujas 20 minutos después?

Solución: Fijemos el origen en el punto 12 del reloj y fijemos el sentido horario. La aguja de la hora, cada hora, avanza $30^{\circ}$, con lo que en 20 minutos avanzará $10^{\circ}$ y se situará en la posición de $90^{\circ}+10^{\circ}=100^{\circ}$. La aguja de los minutos, cada hora avanza $360^{\circ}$, con lo que en 20 minutos avanzará $120^{\circ}$, que será su posición al partir del origen. Por lo tanto, a las tres y veinte minutos las agujas formarán un ángulo agudo de $\mathbf{2 0}^{\circ}$, estando ya por delante la aguja de los minutos.

Los problemas de la tercera prueba, que se realizó el 28 de mayo de 2012, y sus respectivas soluciones fueron:

1. En la figura de la derecha vemos un molinillo de viento formado por un cuadrado y cuatro triángulos

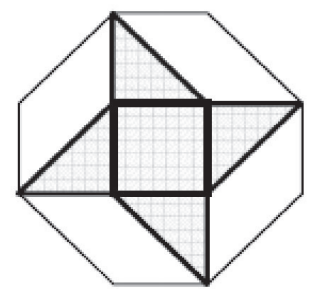


rectángulos isósceles, que está inscrito en un octógono. Si el área del octógono es de $42 \mathrm{~m}^{2}$, hallar el área del molinillo.

Solución: La figura se puede dividir fácilmente en 14 triángulos rectángulos. El área de cada uno de ellos será de $42: 14=3 \mathrm{~m}^{2}$, con lo que el área del molinillo será de 6 × $3=\mathbf{1 8} \mathbf{~ m}^{2}$.

2. Un camionero presupuesta cierta cantidad de dinero para el gasto de carburante en un recorrido de $600 \mathrm{~km}$. Sin embargo, una rebaja repentina en el precio del gasóleo le supuso una rebaja de 0,14 € por $\mathrm{km}$, lo que le permitiría hacer un recorrido de $750 \mathrm{~km}$ con el mismo gasto. Calcular la cantidad que fue presupuestada inicialmente para el carburante.

Solución: El camionero ha conseguido un ahorro total de $600 \times 0,14=84 €$. Con ese dinero, ha podido recorrer $150 \mathrm{~km}$ más. Luego, mediante una simple regla de tres directa, si $150 \mathrm{~km}$ le cuestan $84 €$, los $750 \mathrm{~km}$ le costarán

$$
\frac{750 \times 84}{150}=\mathbf{4 2 0} €
$$

3. Un torneo de tenis se juega por el sistema de "eliminatoria directa", que quiere decir que el perdedor de un partido queda eliminado del torneo. Si en el torneo participan 130 jugadores, ¿cuántos partidos deberán disputarse para conocer al ganador?

Solución: Simplemente hay que darse cuenta de que en cada partido queda eliminado un participante, luego para que haya un ganador deben quedar eliminados 129 jugadores, con lo que deben jugarse 129 partidos.

4. En otro torneo de tenis participaron únicamente 6 jugadores. Cada jugador juega un partido con cada uno de los restantes. Alicia ganó 4 partidos, Beatriz 3, Carlos 2, David 2 y Emilio solo 1. ¿Cuántos partidos ganó Félix?

Solución: Cada participante de este torneo juega con los demás, es decir, habrá un total de $\frac{6 \times 5}{2}=15$ partidos.

Entre los restantes participantes han ganado un total de $4+3+2+2+1=12$, con lo que Felix ganó 3 partidos.

5. Un vendedor ambulante encuentra una oferta y compra pantalones a un precio de $126 €$ la docena y camisetas a un precio $105 €$ cada caja de 20 unidades. Decide comprar 3 docenas de pantalones y 2 cajas de camisetas. En su puesto venderá cada pantalón a 14 y y cada camiseta a $8 €$. Si consigue vender toda la mercancía, ¿cuál será el beneficio que obtenga?

Solución: El vendedor se habrá gastado en total 126 × $3+105$ × $2=588 €$. Si consigue vender toda la mercancía a los precios indicados obtendrá 14 x $36+$ 8 x $40=824 €$, con lo que obtendrá un beneficio de $824-588=\mathbf{2 3 6} €$.

6. Como ya sabes, un número es primo si tiene únicamente dos divisores. Por ejemplo, son números primos el 2, 3, 5, 7, 11, 13, 17, etc. Ahora te pregunto:

a) ¿Cuál es el primer número primo mayor que 200?

b) Pon cinco ejemplos de números que tenga únicamente tres divisores. ¿Qué características cumplen estos números? 
Solución: Es un problema típico de divisibilidad:

a) Si dejamos de lados los números pares que obviamente no son primos, vamos viendo que no son primos ni el 201 (divisible por 3), ni el 203 (divisible por 7), ni el 205 (divisible por 5), ni el 207 (divisible por 3), ni el 209 (divisible por 11). El primer primo mayor de 200 es el 211.

b) Si vamos tanteando, vemos que los primeros números que tienen únicamente 3 divisores son 4, 9, 25, 49, etc. Para que un número tenga únicamente 3 divisores, dicho número debe ser de la forma $p^{2}$, con $p$ un número primo, ya que en ese caso los divisores serán $1, p$ y $p^{2}$.

7. Completa las casillas que faltan de todas las formas posibles:

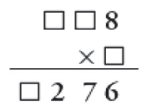

Solución: En la tabla del 8, los únicos que acaban en 6 son 8 x $2=16$ y $8 \times 7=56$. Veamos por donde vamos en el primer caso. Para que la cifra de las decenas del resultado sea un 7 , tendremos que tener en las decenas del primer número un 3 o un 8 . Pero, si fuese 8, nos llevaríamos 1, y luego no podría quedar en las centenas del resultado un 2 . Con lo que las decenas del primer número deben ser un 3 y las centenas del primer número deben ser o un 1 o 6. Los casos posibles serían entonces:

$$
\begin{aligned}
& 138 \times 2=0276 \\
& 638 \times 2=1276
\end{aligned}
$$

Ahora supongamos que multiplicamos por 7. Como nos llevamos 5, al multiplicar las decenas nos tiene que dar un número que acabe en 2. Esto es posible si las decenas del primer número son 6. En este caso nos llevaríamos 4 y tenemos que buscar unas centenas del primer número que al multiplicarlas por 7 den un número acabado en 8 , lo cual solo es posible si el número es 4 . Con lo cual, la única posibilidad que tenemos en este caso es:

$$
468 \times 7=3276
$$

9. Intenta, utilizando únicamente cuatro cuatros y las operaciones fundamentales (suma, resta, multiplicación, división, raíz cuadrada, potencia y paréntesis), formar todos los números de 0 al 10. El primero te lo doy hecho de cuatro formas distintas: $0=4+4-4-4=44-44=4^{4}-4^{4}=\sqrt{4 \times 4}-\sqrt{4 \times 4}$

Solución: Hay varias posibilidades. Unas posibles respuestas serían:

$$
\begin{aligned}
& 1=(4 \times 4):(4 \times 4) \\
& 2=(4 \times 4):(4+4) \\
& 3=(4+4+4): 4 \\
& 4=((4 \times 4)+4): 4 \\
& 5=4^{4-4}+4
\end{aligned}
$$

